\title{
Effects of three-dimensional band structure in angle- and spin-resolved photoemission from half-metallic $\mathrm{La}_{2 / 3} \mathrm{Sr}_{1 / 3} \mathrm{MnO}_{3}$
}

\author{
J. Krempaský, * V. N. Strocov, L. Patthey, P. R. Willmott, R. Herger, and M. Falub \\ Paul Scherrer Institut, CH-5232 Villigen-PSI, Switzerland \\ P. Blaha \\ Institute for Materials Chemistry, TU Vienna, A-1060 Vienna, Austria \\ M. Hoesch \\ European Synchrotron Radiation Facility, 38043 Grenoble, France \\ V. Petrov \\ St. Petersburg State Polytechnical University, St. Petersburg 195251, Russia
}

M. C. Richter, O. Heckmann, and K. Hricovini

Université de Cergy-Pontoise, 95031 Cergy Pontoise Cedex, France

(Received 5 December 2007; published 14 April 2008)

\begin{abstract}
We present an angle- and spin-resolved photoemission study of half-metallic three-dimensional perovskite $\mathrm{La}_{2 / 3} \mathrm{Sr}_{1 / 3} \mathrm{MnO}_{3}$ thin films grown with pulsed laser deposition. The experiments are performed in the (100) mirror plane. The experimental results are related to GGA $+U$ calculations through simulations of photoemission spectra, which take into account the final state broadening in surface-perpendicular momentum and the initial-state broadening in energy. We demonstrate that due to the three-dimensional nature of $\mathrm{La}_{2 / 3} \mathrm{Sr}_{1 / 3} \mathrm{MnO}_{3}$, these intrinsic mechanisms of the photoemission process give rise to deviations of the photoemission spectrum from the spectral function corresponding to the strict momentum conservation. In the spin-integrated data, we identify ghost spectral intensity coming from states with different momenta and, in particular, nonvanishing intensity filling the whole interior of the central Fermi surface spheroid. In the spin-resolved data, we find experimental polarization values near the Fermi surface of 55\% at the normal emission and $80 \%$ at off-normal emission. Despite a seeming contradiction to the half-metallic picture, such a reduction in spin contrast is reproduced by our simulations as a result of the broadening mechanisms and is consistent with the half-metallic nature of $\mathrm{La}_{2 / 3} \mathrm{Sr}_{1 / 3} \mathrm{MnO}_{3}$.

DOI: 10.1103/PhysRevB.77.165120

PACS number(s): 71.18.+y, 71.38.-k, 75.47.Lx
\end{abstract}

\section{INTRODUCTION}

Among the Mn oxide compounds, the exotic physics of mixed-valence manganites $\mathrm{La}_{1-x} \mathrm{Sr}_{x} \mathrm{MnO}_{3}$ (LSMO) have received particular attention. Besides the famous colossal magnetoresistive (CMR) behavior, a notion of "half-metallic ferromagnets" is often cited for LSMO compounds, which is an important issue for spintronics applications. Below the Curie temperature $\left(T_{c}\right)$ the hole-doped $\mathrm{La}_{2 / 3} \mathrm{Sr}_{1 / 3} \mathrm{MnO}_{3}$ is a doubleexchange ferromagnet exhibiting symbiotic ferromagnetism and metallic conductivity through $\mathrm{Mn}^{3+}$ and $\mathrm{Mn}^{4+}$ valence states. The ground state of these electrons, subject to a strong Hund's rule coupling to the localized $t_{2 g}$ spins, is predicted to be $100 \%$ spin polarized. ${ }^{1}$ The most direct method to measure spin polarization is based on photoemission (PE) because this technique directly probes the spin polarization of the occupied electronic states. In the pioneering work of Park et al., ${ }^{2}$ a metallic cutoff at the Fermi energy $\left(E_{F}\right)$ for the majority spins and an insulating gap of $600 \mathrm{meV}$ for the minority spins (half-gap) was observed. This evidence of $100 \%$ spin polarization is in contrast to spin polarization data based on transport measurements (see the review of Nadgorny $^{3}$ ), which generally do not allow one to measure $100 \%$ spin polarization. For this reason, $\mathrm{La}_{2 / 3} \mathrm{Sr}_{1 / 3} \mathrm{MnO}_{3}$ was proposed to be a transport half-metal rather than a conventional (true) half-metal, where the minority density of states (DOS) is at $E_{F} N_{\downarrow}\left(E_{F}\right)=0$. The absolute spin polarization measured in Ref. 2 remains subject of debate with spin polarizations ranging from $90 \%$ (Ref. 4) to $35 \%$ (Ref. 3), suggesting that there must be some contribution of the minority spin bands at $E_{F}\left[N_{\downarrow}\left(E_{F}\right) \neq 0\right]$. Thus, there has been continued experimental effort over several years to convincingly verify the truly half-metallic $100 \%$ polarization at the Fermi level.

An important limitation of the PE-based measurements by Park et al. ${ }^{2}$ was the fact that they were unresolved in $\mathbf{k}$ space. Namely, the surface roughness of their sample precluded a defined crystallographic direction, and so their measurements had to be considered angle integrated. Improved sample quality with a well-defined surface orientation and monocrystalline film growth are central issues for successful angle-resolved PE spectroscopy (ARPES) measurements on $\mathrm{La}_{2 / 3} \mathrm{Sr}_{1 / 3} \mathrm{MnO}_{3}$.

$\mathrm{La}_{2 / 3} \mathrm{Sr}_{1 / 3} \mathrm{MnO}_{3}$ is a three-dimensional (3D) system. Its electronic structure near $E_{F}$ is formed by two $e_{g}$ orbitals having $3 z^{2}-r^{2}$ and $x^{2}-y^{2}$ symmetries (see, for example, a review in Ref. 5). The Fermi surface (FS) consists of an electron spheroid around the $\Gamma$ point in the Brillouin zone 
(a)

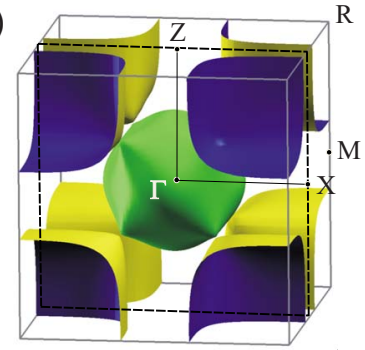

(b)

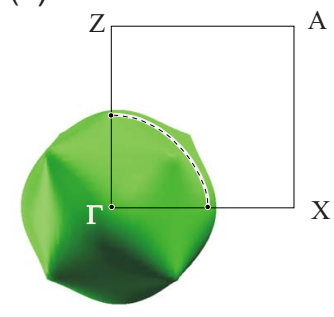

FIG. 1. (Color online) (a) GGA $+U$ calculated Fermi surface of $\mathrm{La}_{2 / 3} \mathrm{Sr}_{1 / 3} \mathrm{MnO}_{3}$. (b) Cut (dashed line) of the central FS spheroid in the (100) mirror plane ( $\Gamma X$ azimuth) where our ARPES and SARPES spectra were measured.

(BZ) center, and hole cuboids around the $R$ points in the BZ corners, as shown in Fig. 1. Such a FS, also shown in Ref. 6, has a pronounced $3 \mathrm{D}$ character. ARPES is a power technique to determine the electron dispersions $E(\mathbf{k})$ with resolution in $\mathbf{k}$ space. However, the determination of 3D dispersions suffers from a fundamental property of the ARPES experiment that due to inelastic scattering the extension of the PE final state wave function into the crystal interior is limited to the mean free path $\lambda$ the order of a few angstroms. Such a confinement in the surface-perpendicular coordinate results in an intrinsic uncertainty in the surface-perpendicular $k_{\perp}$ momentum, described by its broadening $\delta k_{\perp}$ in the order of a few tenths of the BZ extension. This gives rise to a significant distortion in the PE signal from the bands with dispersion in $k_{\perp}$ (see, for example, a recent review in Ref. 7 and the references therein).

Here, we present angle- and spin-resolved PE data on $\mathrm{La}_{2 / 3} \mathrm{Sr}_{1 / 3} \mathrm{MnO}_{3}$ concentrating on the central spheroid of the FS in Fig. 1(b). The experimental results are supported by band calculations and simulations of the ARPES spectra, taking $k_{\perp}$ broadening into account. We demonstrate that the $3 \mathrm{D}$ nature of $\mathrm{La}_{2 / 3} \mathrm{Sr}_{1 / 3} \mathrm{MnO}_{3}$, in combination with $k_{\perp}$ broadening, gives rise to a plethora of unusual effects, which may look like a collapse of the interpretation of the ARPES data in terms of direct transitions in photoemission. In particular, in our spin-resolved data, the 3D effects appear as a reduction in spin contrast at the FS, which might otherwise be misinterpreted as deviations of $\mathrm{La}_{2 / 3} \mathrm{Sr}_{1 / 3} \mathrm{MnO}_{3}$ from the half-metallicity.

\section{EXPERIMENT AND RESULTS}

Our measurements were made on a $130 \mathrm{~nm}$ thick holedoped $\mathrm{La}_{2 / 3} \mathrm{Sr}_{1 / 3} \mathrm{MnO}_{3}$ film deposited on a $\mathrm{SrTiO}_{3}(001)$ substrate by pulsed laser deposition. ${ }^{8}$ The sample showed a clear $1 \times 1$ low energy electron diffraction pattern without signs of surface reconstructions. The measured $T_{C}=297 \mathrm{~K}$, in coincidence with the maximum of the sample resistivity, was typical of these CMR materials. The sample was in situ transferred to the ARPES spectrometer at the Surface and Interface Spectroscopy Beamline at the Swiss Light Source at Paul Scherrer Institut for the ARPES measurements. The electric field vector of the linearly polarized synchrotron light and the sample normal were in the plane of incidence,

with the incidence angle being $45^{\circ}$ relative to the sample normal. The sample was oriented in such a way that the (100) mirror plane of the crystal ( $\Gamma X$ azimuth) was in the plane of incidence. During the experiment, the sample temperature was $60 \mathrm{~K}$. The photon energy $h \nu=38 \mathrm{eV}$ was chosen to maximize the normal emission intensity at $E_{F}$, which corresponds to the Fermi surface crossing along the $\Gamma Z$ direction of the BZ. 9

Figure 2 shows our spin-integrated data: (a) the energy distribution curves (EDCs) and (b) the momentum distribution curves (MDCs) rendered from them in the experiment and $[(\mathrm{c})$ and (d)] ARPES simulations discussed later. The raw EDC plots were normalized to the background above $E_{F}$. The energy resolution of the analyzer was $50 \mathrm{meV}$. In order to see more clearly the experimental dispersive feature of the spectral features $\mathrm{A}$ and $\mathrm{B}$, the second derivative of the ARPES spectra in Figs. 2(a) and 2(b) is plotted in color (e) such that light parts correspond to energy bands. Similarly for the simulated data, the second derivative ARPES is seen in (f).

The spin-resolved ARPES (SARPES) measurements on the in situ transferred sample were performed at the same beamline using the complete photoemission experiment (COPHEE) spectrometer equipped with two Mott detectors. ${ }^{10}$ As the spin detection efficiency of the Mott detectors is a factor of 1000 smaller compared to that of the spin-integrating analyzer, the resolution in the spin-resolved photoemission was relaxed to $150 \mathrm{meV}$. Since the epitaxial $\mathrm{La}_{2 / 3} \mathrm{Sr}_{1 / 3} \mathrm{MnO}_{3}$ films have an in-plane magnetic anisotropy with the $\langle 110\rangle$ easy magnetization axis, ${ }^{11}$ the sample was in situ magnetized with a pulsed field of $\sim 100$ Oe parallel to the sample surface in the [110] direction ( $\Gamma M$ azimuth). As revealed by ex situ magnetic hysteresis measurements, this pulsed magnetic field was sufficiently intense to magnetize the sample remanently. In order to eliminate the instrumental asymmetry of the Mott detectors (Sherman function of 13\%), the data were cross-checked by magnetizing the sample in opposite directions. The incident geometry, azimuthal orientation, and photon energy $h \nu=38 \mathrm{eV}$ were the same as for the above ARPES experiment. The measurements were performed for two emission angles, corresponding to the normal emission and to $k_{\|}=0.4 \pi / a$ at the binding energies near $E_{F}$.

The raw SARPES measurements of the Mott detector counts $N^{+}$and $N^{-}$were first rendered into the in-plane and out-of-plane components of the spin polarization $P=\frac{N^{+}-N^{-}}{N^{+}+N^{-}}$ shown in Figs. 3(a) and 3(b). The measured polarization is found to be parallel to the easy magnetization axis along the $\Gamma-M$ direction (no out-of-plane spin polarization is detected), which is in agreement with the in-plane magnetization in epitaxial (strained) $\mathrm{La}_{2 / 3} \mathrm{Sr}_{1 / 3} \mathrm{MnO}_{3}$ films. Second, based on $P$ and the total yield of the Mott detectors $N^{+}+N^{-}$, the majority $\left(I_{\uparrow}\right)$ and minority $\left(I_{\downarrow}\right)$ currents were calculated [Figs. 3(c) and 3(d)].

Our SARPES results show significant differences from the essentially angle-integrated results by Park et $a .^{2}$

(i) Our values of spin polarization at $E_{F}$ are $55 \pm 10 \%$ for $k_{\|}=0$ and $80 \pm 10 \%$ for $k_{\|}=0.4 \pi / a$. This is much lower than the values close to $100 \%$ reported by Park et al.

(ii) The $P$ spectra in Fig. 3 show distinct features (marked with arrows), which appear as kinks in the $I_{\downarrow}$ plots (see the 

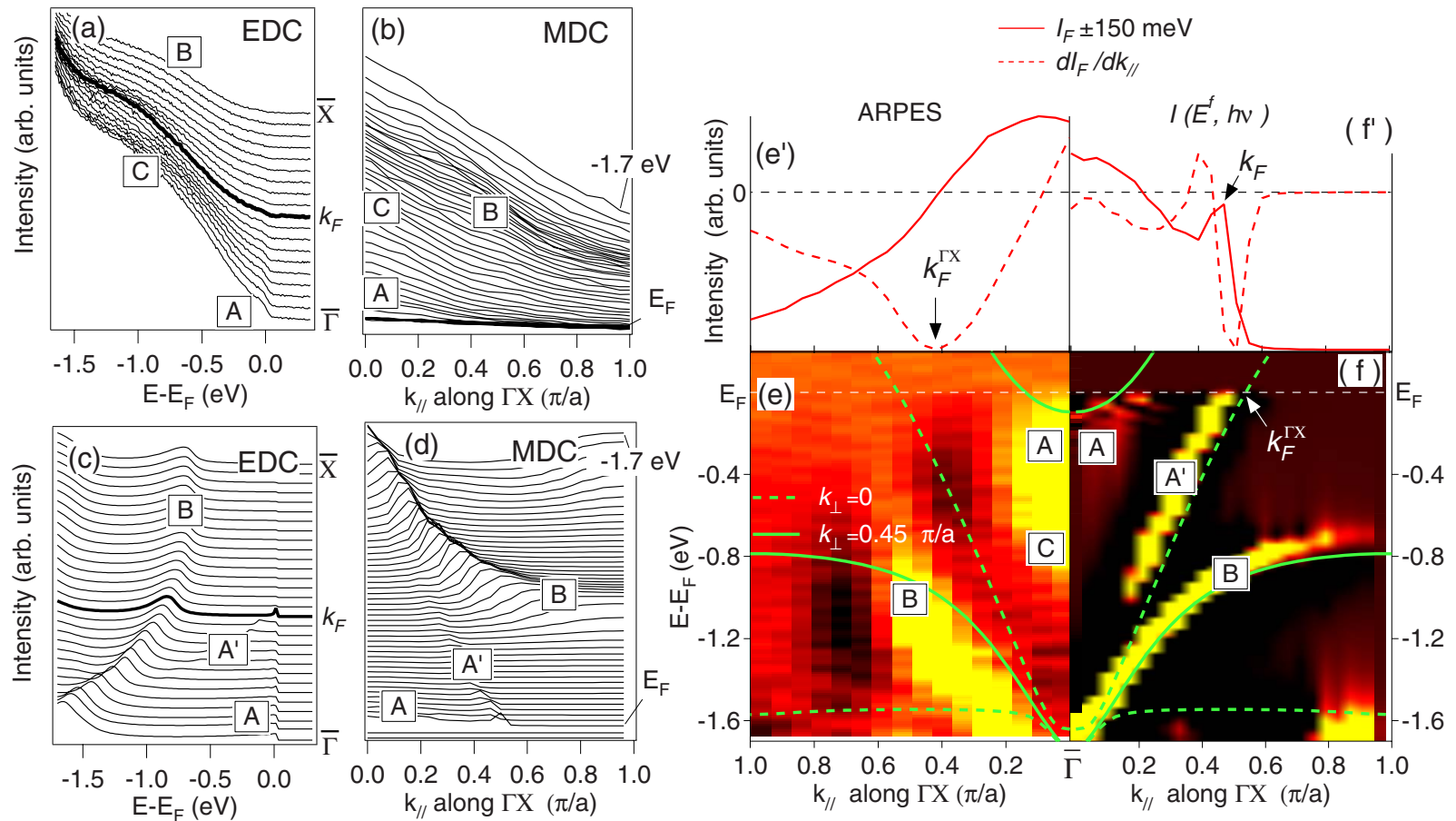

FIG. 2. (Color online) Experimental spin-integrated (a) EDC and (b) MDC spectra, measured with $h \nu=38 \mathrm{eV}$, compared to simulated (c) EDC and (d) MDC spectra rendered from ARPES simulations in Fig. 4(b). In (a) and (c), the EDC corresponding to $k_{F}$ is shown in bold. (e) The negative second derivative of the experimental ARPES data (yellow=maximum amplitude) is compared to that of the simulated ARPES intensity map in (f). [( $\left.\mathrm{e}^{\prime}\right)$ and $\left.\left(\mathrm{f}^{\prime}\right)\right]$ The Fermi intensity $I_{F}$ (full line) is integrated $\pm 150 \mathrm{meV}$ at $E_{F}$ and is compared for both experiment (e') and simulation $\left(\mathrm{f}^{\prime}\right)$ together with their first derivative plots (dashed lines). The band dispersions at $k_{\perp}=0$ include the conduction band bottom [full lines in (e) and (f)] and those at $k_{\perp}=0.45 \pi / a$ corresponding to $h \nu=38 \mathrm{eV}$ (dashed lines). The spectral features A, A', B, C, and Fermi momenta $k_{F}^{\Gamma X}$ on both experimental and theoretical sides are discussed in the text.

insets). More specifically, for $k_{\|}=0.4 \pi / a$, there is a sharp onset in $I_{\downarrow}$ near a binding energy of $200 \mathrm{meV}$. For $k_{\|}=0$, there is less pronounced but still notable onset near $400 \mathrm{meV}$. These features are missing in the data of Park et al.

(iii) Consistent with the spin-integrated data, our $I_{\uparrow}$ and $I_{\downarrow}$ spectra show a broad spectral feature " $C$ " in the region of $1 \mathrm{eV}$. For $k_{\|}=0$, this feature is more pronounced and notably shifted to lower energies in $I_{\uparrow}$ compared to $I_{\downarrow}$. For $k_{\|}=0.4 \pi / a$, it becomes less pronounced in both $I_{\uparrow}$ and $I_{\downarrow}$. No such structures were observed by Park et al.

\section{THEORETICAL MODEL}

\section{A. Band calculations}

The experimental spectra were interpreted based on generalized gradient approximation with Hubbard parameter $(\mathrm{GGA}+U)$ band calculations. To account for Sr doping, the calculations were performed within the virtual crystal approximation (VCA). This approximation replaces the random crystal potential with intermixed $\mathrm{La}$ and $\mathrm{Sr}$ atoms by a periodic one taken as an average of the $\mathrm{La}$ and $\mathrm{Sr}$ atomic potentials. Although the VCA leaves away the effects of broadening in $\mathbf{k}$ space associated with disorder, it is presently the only numerically feasible method to treat complex disordered systems such as LSMO. Furthermore, our study focuses on the $\mathrm{Mn}-\mathrm{O}$ derived $e_{g}$ states near $E_{F}$ where the dis-
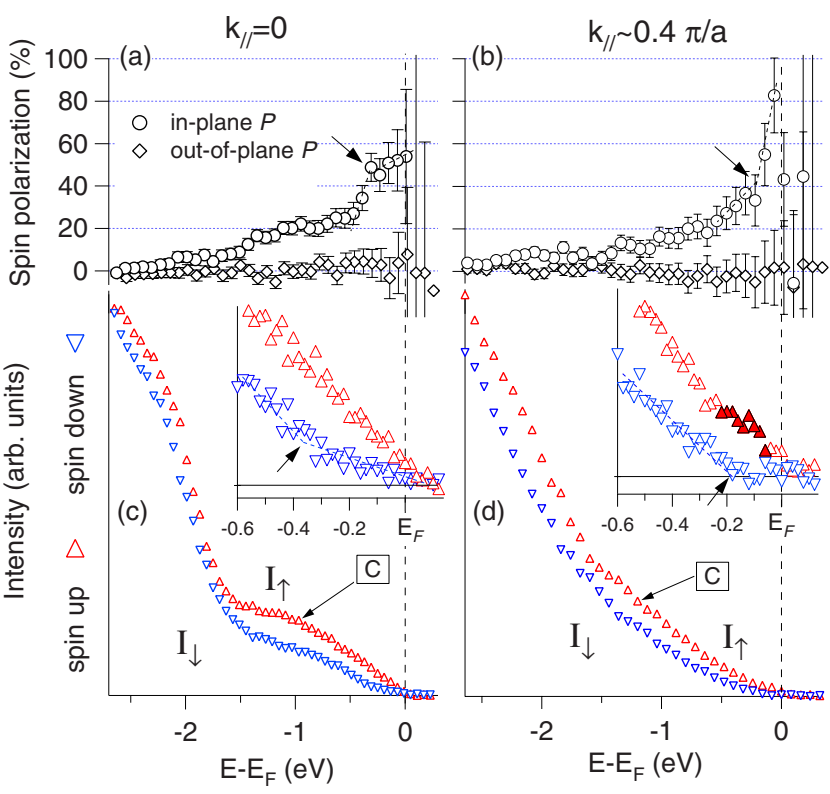

FIG. 3. (Color online) $[(\mathrm{a})$ and (b)] Spin polarization $P$ measured with $h \nu=38 \mathrm{eV}$. The error bars reflect statistical errors of $N^{+}$ and $N^{-}$electron count rates. [(c) and (d)] Spin-resolved spectra for $I_{\uparrow}$ and $I_{\downarrow}$ for $k_{\|}=0,0.4 \pi / a$. Data in the insets have $\Delta E=0.02 \mathrm{eV}$; in other panels, they are averaged over $\Delta E=0.08 \mathrm{eV}$. Dashed lines are a guide to the eye that highlight kinks in $I_{\downarrow}$ marked by arrows. The $I_{\uparrow}$ shoulder, marked with $\boldsymbol{\Delta}$ in inset (b), is discussed in the text. 
(a)

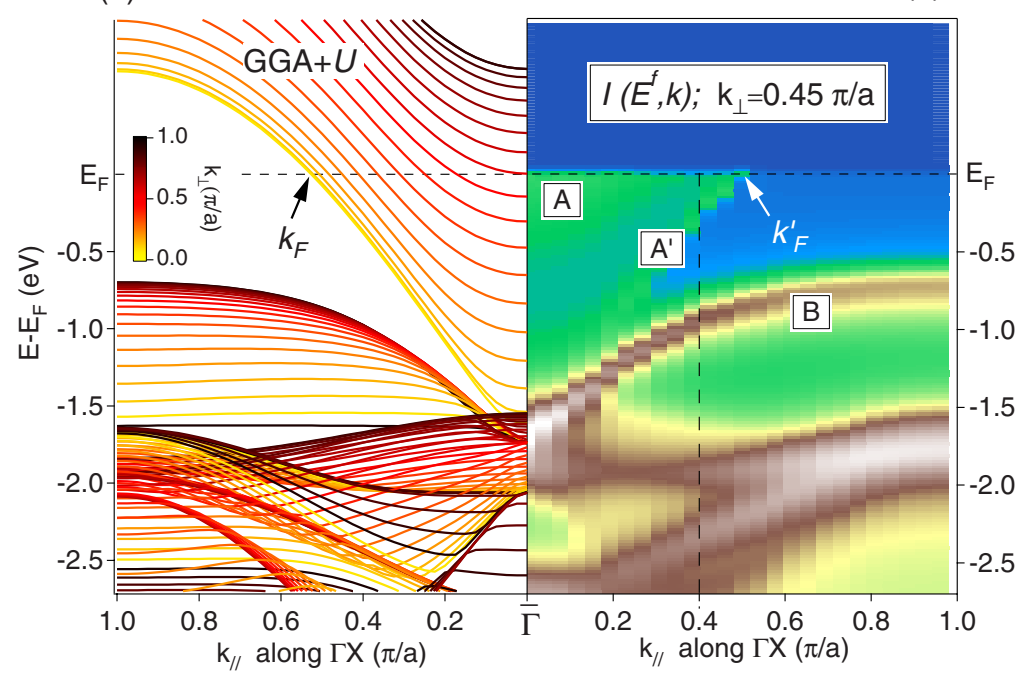

minority spin

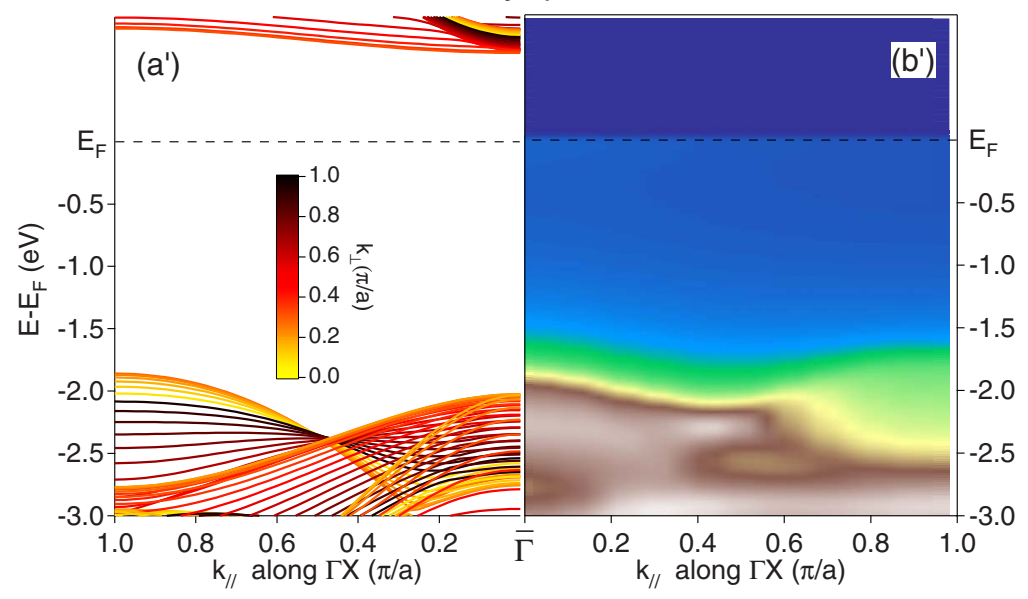

FIG. 4. (Color online) $k_{\|^{-}}$and $k_{\perp}$-resolved $\mathrm{GGA}+U(U=2 \mathrm{eV})$ dispersions of the (a) majority and $\left(\mathrm{a}^{\prime}\right)$ minority spin bands along $\Gamma X$. The simulations of the ARPES intensity maps corresponding to $k_{\perp}^{0} \sim 0.45 \pi / a(h \nu=38 \mathrm{eV})$ are seen in (b) for majority and in $\left(b^{\prime}\right)$ for minority spin bands. The spectral features $\mathrm{A}, \mathrm{A}^{\prime}$, and $\mathrm{B}$ are discussed in the text, $k_{F}$ and $k_{F}^{\prime}$ are Fermi momenta $\left(k_{F}^{\prime}<k_{F}\right)$, and the vertical dashed line in (b) corresponds to the off-normal-emission angle where spin-resolved data in Fig. 3(b) are presented. order effects are less pronounced for the following reasons: (1) In the LSMO structure, the $\mathrm{MnO}$ planes are separated from the disordered $\mathrm{LaSrO}$ planes; (2) despite different atomic radii, the scattering potential of $\mathrm{Sr}$ and $\mathrm{La}$ atoms for the valence electrons is similar, which results in a fairly small perturbation of the valence band electronic structure. A tetragonal distortion of the LSMO perovskite structure with an axial ratio $c / a$ of $\sim 0.99$ was used to account for a slight tetragonal distortion of the epitaxial films. ${ }^{8}$ The calculations were performed with the WIEN $2 K$ code $^{12}$ employing the augmented plane wave plus local orbital (APW+LO) computational scheme.

The $U$ parameter in our calculations was adjusted to the experimental data and cannot be directly related to electron correlations like Hubbard's $U$. We have estimated the $U$ parameter to be around $2 \mathrm{eV}$ from the energy position of the $t_{2 g}$ states, which were most sensitive to its variations. The majority $e_{g}$ states were fairly insensitive to $U$ and already formed the correct FS with $U=0$. However, having $U>0$ is essential to reproduce the half-metallic properties of $\mathrm{La}_{2 / 3} \mathrm{Sr}_{1 / 3} \mathrm{MnO}_{3}$ because with $U=0$ the minority $e_{g}$ states reach the Fermi level. In fact, the effect of $U$ on the band structure has been addressed by several authors. ${ }^{14}$ Livesay et al. ${ }^{6}$ noted that there are two small minority electron sheets at $E_{F}$ and that a tiny downward shift in the Fermi energy would make these disappear, causing the system to become half-metallic. We note that this is exactly what happens also for GGA $+U$ calculations, with $U \neq 0$. Interestingly, the previous calculations on layered LSMO in Ref. 13 also used $U=2 \mathrm{eV}$ and showed qualitatively the same effect of $U$ on the spin-up and spin-down bands.

Computational results in the (100) mirror plane for the spin-up band structure are shown in Figs. 4(a) and 4(b) as a projection of the 3D dispersions $E\left(k_{\|}, k_{\perp}\right)$, where $k_{\perp}$ is displayed by means of a color scale ranging from yellow for $k_{\perp}=0$, through red to black for $k_{\perp}=|\Gamma Z|$. The bands with different $k_{\perp}$ momenta cross $E_{F}$ at different $k_{\|}$. Here, we focus on the central 3D paraboloid around the $\Gamma$ point, which forms the central electron spheroid of the FS. The $k_{\perp}$ dispersion of the paraboloid is due to the $3 z^{2}-r^{2}$ orbital contribution to the wave function. The band just below the paraboloid is formed from other $e_{g}$ bands derived from the $x^{2}-y^{2}$ orbital.

The horizontal sections of the FS shown in Fig. 1 are formed by bands having strong $k_{\perp}$ dispersion due to the large weight of the $3 z^{2}-r^{2}$ orbital in their wave function. On the other hand, the vertical sections with negligible $k_{\perp}$ dispersion are formed by an overwhelming weight of the $x^{2}-y^{2}$ 


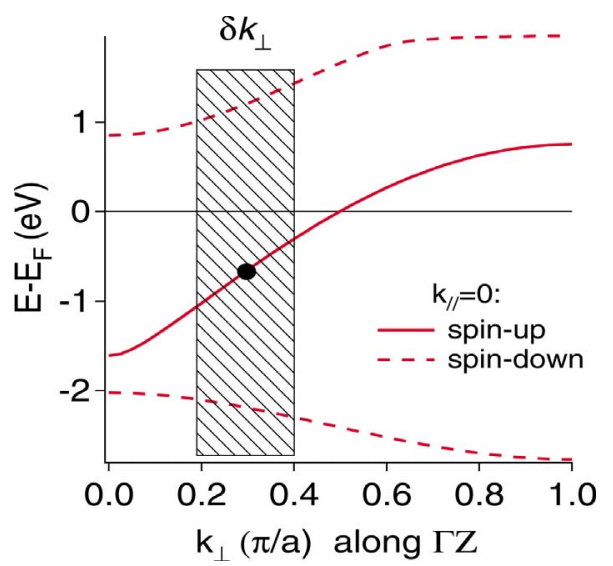

FIG. 5. (Color online) Spin-resolved GGA $+U$ band structure dispersions along the $\Gamma Z$ direction in normal emission $\left(k_{\|}=0\right)$. Due to $k_{\perp}$ broadening, for a given $k_{\perp}^{0}(\bullet)$, the spectral intensity $I\left(E^{f}, h \nu\right)$ in ARPES simulations for low photon energies is averaged over $\delta k_{\perp}$ equivalent to $\sim 20 \%$ of the BZ (hatched area).

orbital. Thus, the ARPES and SARPES study in the $\Gamma-X$ azimuth presented in this paper is sensitive to bands with the $3 z^{2}-r^{2}$ orbital character.

\section{B. Photoemission simulations: Lifetime and $k_{\perp}$-broadening effects}

A fundamental difficulty in the interpretation of ARPES data on three-dimensional systems is an intrinsic broadening of the electron momentum component perpendicular to the crystal surface $k_{\perp}$. This results from the fact that due to a finite photoelectron lifetime, the final state extension perpendicular to the surface is confined by the photoelectron escape depth $\lambda$. The intrinsic broadening is then inversely proportional to it as $\delta k_{\perp}=1 / \lambda$, with $\delta k_{\perp}$ taken at full width at half maximum (FWHM).

Simulations of the ARPES spectra including both finite photoelectron and photohole lifetimes were performed as described in Ref. 7. Briefly, the final-state (photoelectron) spectral function $A^{f}(E, \mathbf{k})$ is reduced in energy to the $\delta$ function at the fixed final-state energy $E^{f}$. However, due to finite photoelectron lifetime, $A^{f}(E, \mathbf{k})$ has an intrinsic broadening in $k_{\perp}$ characterized by the Lorentzian distribution $A^{f}\left(k_{\perp}\right)$ $=\frac{\delta k_{\perp}}{\left(k_{\perp}-k_{\perp}^{0}\right)^{2}-\left(\delta k_{\perp} / 2\right)^{2}}$. This distribution is centered at $k_{\perp}^{0}$ of the nominal direct transition given by the final-state dispersion at $E^{f}$ and has FWHM equal to $\delta k_{\perp}$. This is schematically depicted in Fig. 5, where for a given $k_{\|}^{0}=0(\bullet)$, the spectral intensity $I\left(E^{f}, h \nu\right)$ is to be weighted over the $\delta k_{\perp}$ interval (hatched area).

On the other hand, the initial-state (photohole) spectral function $A^{i}(E, \mathbf{k})$ is reduced to the $\delta$ function at a welldefined $k_{\perp}$ characteristic of propagating Bloch waves. However, due to the finite photohole lifetime, it has an intrinsic broadening in energy characterized by the Lorentzian distribution $A^{i}(E)=\frac{\delta E}{\left(E-E^{i}\left(k_{\perp}\right)\right)^{2}-(\operatorname{Im} \Sigma)^{2}}$. Contrary to the $k_{\perp}$ broadening mentioned above, this distribution is centered at the quasiparticle (QP) band energy $E^{i}\left(k_{\perp}\right)$ and has a lifetime broadening at FWHM given by the imaginary part of the electron self-energy as $2 \operatorname{Im} \Sigma$. It should be noted that the Lorentzian shape of $A^{i}(E)$ is, in fact, a zero-order approximation, implying that $\operatorname{Im} \Sigma$ is energy independent. ${ }^{15}$ As shown below, such a correction to $A^{i}(E)$ in the simulated spectral function $I\left(E^{f}, h \nu\right)$ hardly affects the analysis of our ARPES data.

Assuming a constant photoemission matrix element, the ARPES signal for a given $E^{f}$ and photon energy $h \nu$ is then found by the integration of the above spectral functions over $k_{\perp}$ through the whole BZ as $I\left(E^{f}, h \nu\right)=\int_{B Z} A^{f}\left(k_{\perp}\right) A^{i}(E) d k_{\perp}$. The initial-state spectral function thus appears in the ARPES spectrum as weighted over the final-state $k_{\perp}$ broadening interval around the nominal direct transition at $k_{\perp}^{0}$.

In such a case, a correct description of the final states would require calculations on top of the APW $+\mathrm{LO}$ of complex band structure and matching of the damped Bloch waves to the outgoing photoelectron plane wave in vacuum. At the present computational level, this strict approach is hardly feasible for complex systems such as LSMO, which has forced us to use the free-electron approximation for the final states, completely ignoring the matrix elements.

The main parameter of the simulations, i.e., the $\delta k_{\perp}$ finalstate broadening, is determined by the photoelectron escape depth $\lambda$. As no experimental data on $\lambda$ are available for $\mathrm{La}_{2 / 3} \mathrm{Sr}_{1 / 3} \mathrm{MnO}_{3}$, we have estimated it from the "universal curve." In our photon energy region, it predicts $\lambda$ of the order of $5 \AA$, which translates into $\delta k_{\perp}$ about $\sim 20 \%$ of the BZ (Fig. 5).

Another simulation parameter, i.e., the $\operatorname{Im} \Sigma$ initial-state lifetime broadening, was estimated from the experimental spectral peaks originating from the bands flat in $k_{\perp}$, whose energy broadening is reduced to $2 \operatorname{Im} \Sigma$. A reasonable description was found with $\operatorname{Im} \Sigma$ varying linearly from zero at $E_{F}$ to $0.5 \mathrm{eV}$ at a binding energy of $8 \mathrm{eV}$ near the valence band bottom [the region of the quadratic energy dependence near $E_{F}$ typical of the Fermi liquid (FL) is negligible on the valence band energy scale]. Interestingly, this energy dependence of $\operatorname{Im} \Sigma$ appears to be close to that found from ARPES on a series of noble materials. ${ }^{16}$ Finally, we have incorporated the finite experimental resolution by convoluting the simulated spectra with a Gaussian having the corresponding FWHM of $50 \mathrm{meV}$ for the spin-integrated ARPES and of $150 \mathrm{meV}$ for the spin-resolved ARPES.

For comparison with our experimental spin-integrated data obtained with variable $h \nu$, we have performed ARPES simulations through the whole $k_{\|}$extension of the $\mathrm{BZ}$ with a discrete set of the $k_{\perp}^{0}$ momenta along the $\Gamma Z$ direction. The full results of these simulations are available as a movie. ${ }^{17} \mathrm{~A}$ few important excerpts follow.

1. The simulated intensity at $E_{F}$ for $k_{\|}=0$ is plotted in Fig. 7(b), full line. Interestingly, due to $k_{\perp}$ broadening, the maximum of intensity at $E_{F}$ appears at around $k_{\perp}^{0}=0.45 \pi / a$, which is notably displaced from the exact theoretical Fermi vector $k_{F}=0.49 \pi / a$. In the following, such wave vectors delivering maximal photoemission intensity at $E_{F}$ will be designated as $k_{F}^{\prime}$.

2. The $k_{\|}$dispersion map of the spectra simulated with the above $k_{\perp}^{0}=k_{F}^{\prime}$ at the Fermi intensity maximum is shown in Fig. 2(f), mirrored with the experimental map in Fig. 2(e). These data are also represented as EDC and MDC plots in Figs. 2(c) and 2(d) below the experimental curves. 


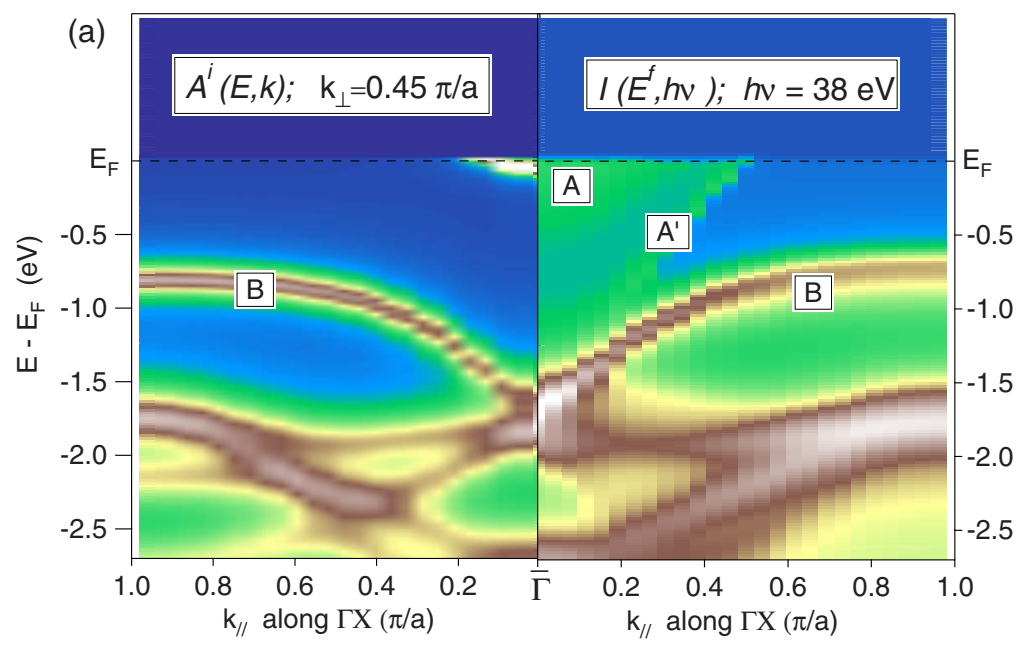

(b)

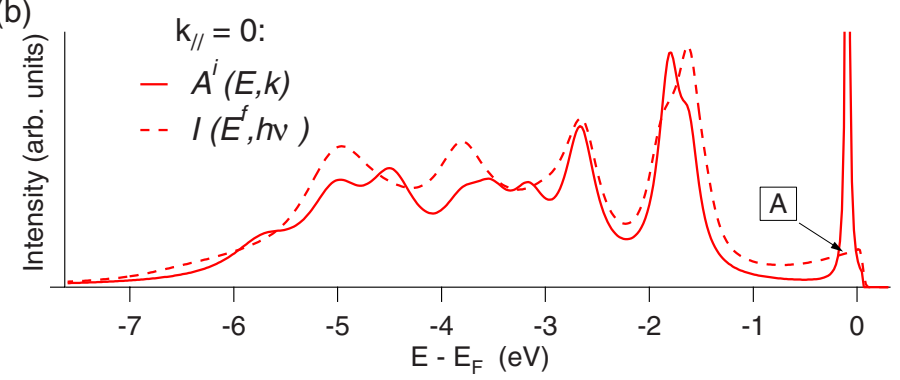

FIG. 6. (Color online) (a) A comparison between the spectral function $A^{f}(E, \mathbf{k})$ (left) and the ARPES spectrum $I\left(E^{f}, h \nu\right)$ with $k_{\perp}$-broadening effects. These effects are pronounced for the $e_{g}$ states, which appear as a filled paraboloid in ARPES. In particular, for $k_{\|}=0$, (b) the spectral function shows a quasiparticle peak close to $E_{F}$, which is quenched to the remnant spectral feature (A) in ARPES.
The simulated $k_{\|}$dispersion maps in Figs. 2(f) and 6 immediately show effects of $k_{\perp}$ broadening, particularly in the region of the $3 z^{2}-r^{2}$ paraboloid: First, without $k_{\perp}$ broadening $\left(\delta k_{\perp}=0\right)$, the ARPES peaks would exactly follow the band structure for the nominal direct transition at $k_{\perp}^{0}=k_{F}^{\prime}$ [full lines in Figs. 2(e) and 2(f)], and the signal from the paraboloid would reduce to a narrow peak close to $E_{F}$ dispersing around $k_{\|}=0$. With $k_{\perp}$ broadening, the ARPES intensity for a given $k_{\|}$picks up contributions from all $k_{\perp}$ through BZ. This results in the appearance of the paraboloid as a sort of projection over $k_{\perp}$ with nonvanishing intensity filling out its whole interior and with only a remnant intensity enhancement near $k_{\|}=0$. In particular, a clear Fermi edge is seen through the whole $k_{\|}$extension of the paraboloid. This spectral feature is labeled A. Second, there appears feature $\mathrm{A}^{\prime}$, which has no counterpart in the band structure for $k_{\perp}^{0}=k_{F}^{\prime}$, but only in that for $k_{\perp}^{0}=0$ [dashed line in Fig. 2(e)]. Since features $\mathrm{A}$ and $\mathrm{A}^{\prime}$ become accessible via $k_{\perp}$ broadening, we label them as "ghost" features. Feature A' corresponds to the minima of the $k_{\perp}$ dispersion of the paraboloid (with a slight intrinsic shift inside the band interior ${ }^{7}$ ), whose contribution to the total intensity is enhanced due to the singularity of one-dimensional DOS $\left(\frac{\partial E}{\partial k_{\perp}}\right)^{-1}$ (1DOS).

Finally, feature $\mathrm{B}$ originates from the $k_{\|}$dispersion of electrons with the $x^{2}-y^{2}$ orbital character. It sticks to the upper edge of the band, which gives the largest contribution to the ARPES intensity because of flat $k_{\perp}$ dispersion and, thus, large 1DOS.

\section{ARPES spectra vs spectral function in three dimensions}

For two-dimensional systems, such as bilayer manganites, the ARPES spectra, neglecting the matrix element variations, directly represent the photohole spectral function $A^{i}(E, \mathbf{k})$. This fact is often used to test the Fermi liquid properties of the electron system in solids. ${ }^{18,19}$ In fact, for the bilayer manganites, the QP spectral function was experimentally observed. $^{20}$

However, for 3D systems, such as in our case, the connection between ARPES and $A^{i}(E, \mathbf{k})$ is more complex. Namely, the ARPES spectrum for a given $k_{\|}$is formed by piling up all $A^{i}(E, \mathbf{k})$ whose $k_{\perp}$ spans over the whole $k_{\perp}$-broadening interval, which, in our case, is $\sim 20 \%$ of the BZ extension. To illustrate this effect, Fig. 6(a) compares the simulated ARPES intensity [also seen in Fig. 4(b)] and the pure spectral function $A^{i}(E, \mathbf{k})$. Figure $6(\mathrm{~b})$ captures the difference between the two for $k_{\|}=0$. It clearly shows that (i) the extraction of $\mathbf{k}$-resolved $A^{i}(E, \mathbf{k})$ for $3 \mathrm{D}$ materials is nontrivial in particular for the $e_{g}$ electron states; (ii) the experimental observation of $\mathrm{QP}$ peaks, smeared in $k_{\perp}$, is more challenging.

\section{DISCUSSION}

\section{A. Spin-integrated ARPES}

The experimental photon energy dependence of the ARPES intensity at $E_{F}$ for $k_{\|}=0$ is shown in Fig. 7(b), with the $h \nu$ values rendered into $k_{\perp}$ using a free-electron approximation for the final states with an empirical inner potential of $10.16 \mathrm{eV}{ }^{9}$ In principle, for complex materials, such as LSMO, such approximation can only be expected to work in a limited energy and $\mathbf{k}$-space region. We have therefore restricted its use to a narrow energy region near the $\Gamma$ point where the inner potential was adjusted. ${ }^{9}$ The relevance of this 

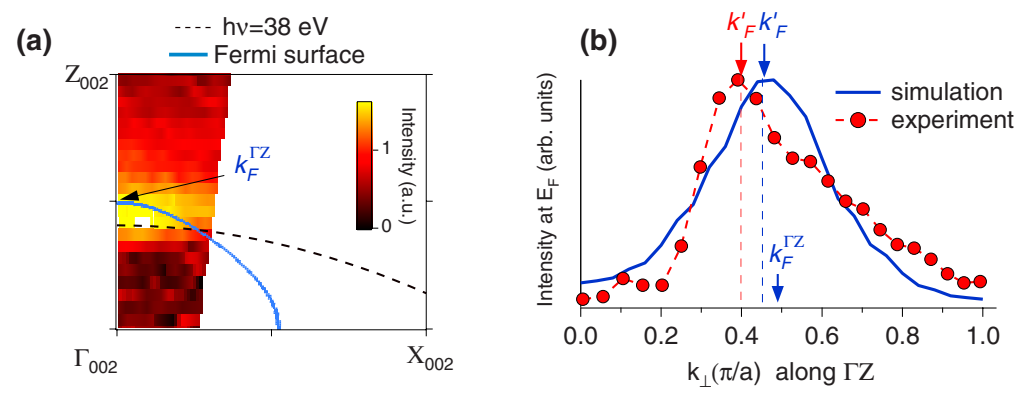

FIG. 7. (Color online) (a) Experimental FS cut in the $\Gamma$ - $X$ azimuth (data taken from Ref. 9). Dashed line represent the path in the $\mathbf{k}$ space of the ARPES measurements with $h \nu=38 \mathrm{eV}$ (free electron final state). The full line represents the FS cut, also seen in Fig. 1(b). (b) Experimental and simulated PE-intensity profile at $E_{F}$ along the $\Gamma Z$ direction $\left(k_{\|}=0\right) . k_{F}^{\Gamma Z}$ denote the theoretical Fermi momentum, which is compared to the experimental one in the text. description of the final states is evidenced in Fig. 7(b) by the agreement of the experimental and theoretical photon energy dependences. The maximum is achieved with $h \nu=38 \mathrm{eV}$, corresponding to $k_{\perp} \sim 0.4 \pi / a$. This experimental dependence is well reproduced by the simulations, although the intensity maximum is shifted to $k_{\perp} \sim 0.45 \pi / a$ (such a minor discrepancy in $k_{\perp}$ can be attributed to inaccuracies of either the free-electron approximation or computations).

The experimental data in Fig. 2 for $h \nu=38 \mathrm{eV}$ should therefore be compared to the simulation for $k_{\perp} \sim 0.45 \pi / a$. The changes of $k_{\perp}$ with variation of $k_{\|}$in our experimental data in Fig. 7(a) are insignificant for our comparison, particularly in view of $k_{\perp}$ broadening.

\section{ARPES spectra vs band structure}

Figure 2 compares the simulated and experimental $k_{\|}$dispersion data sets in various representations. We immediately see the characteristic effects of the $k_{\perp}$ broadening predicted by ARPES simulations, with the nonvanishing intensity filling out the whole interior of the $3 z^{2}-r^{2}$ paraboloid with a clear Fermi edge through its $k_{\|}$extension. In fact, such an effect of the $k_{\perp}$ broadening is fairly typical of 3D materials; for example, it can be recognized in the ARPES spectra of $\mathrm{Cu}$ measured in the region of the $s p$-band paraboloid. ${ }^{21}$ Features A and B readily find their counterparts in the experimental spectra, with convincing agreement on both dispersions and energy positions of these structures, including the position of the conduction band bottom at $\sim 1.7 \mathrm{eV}$. Only feature $\mathrm{A}^{\prime}$ is not visible in the experiment even in the second derivative intensity map in Fig. 2(e). This is presumably because the PE matrix element, ignored in our simulations, normally reduces much in the $k_{\perp}$ dispersion extremes ${ }^{22}$ where feature $\mathrm{A}^{\prime}$ originates.

The line shapes of the experimental and theoretical curves in Fig. 2 indicate, however, a gross discrepancy, the strong and broad structure $\mathrm{C}$ (better resolved in the spin-resolved spectra in Fig. 8). It is centered at $k_{\|}=0$, slightly disperses with $k_{\|}$, and protrudes its MDC tails beyond $k_{\|}$momenta of $0.4 \pi / a$. Dessau and Shen explained a similar structure in a layered LSMO in terms of Franck-Condon broadening (FCB) ${ }^{23}$ Our ARPES simulation does not account for FCB bosonic degrees of freedom in the PE process and, therefore, cannot reproduce this peak. It should be noted that FCB structures identified in ARPES data on layered $\mathrm{LSMO}^{20,23}$ have $k_{\|}$dispersions that are significantly stronger than those in our three-dimensional case. Presumably, the dispersion in $k_{\|}$is suppressed by averaging over $k_{\perp}$ similar to the above $k_{\perp}$-broadening effects for QP peaks.
The dispersion of the experimental band $\mathrm{B}$ in comparison with the GGA $+U$ calculations in Figs. 2(e) and 2(f) does not show any significant renormalization. The situation is, however, different for peak $\mathrm{A}$ around a binding energy of $400 \mathrm{meV}$. In fact, this peak is similar to peak A around
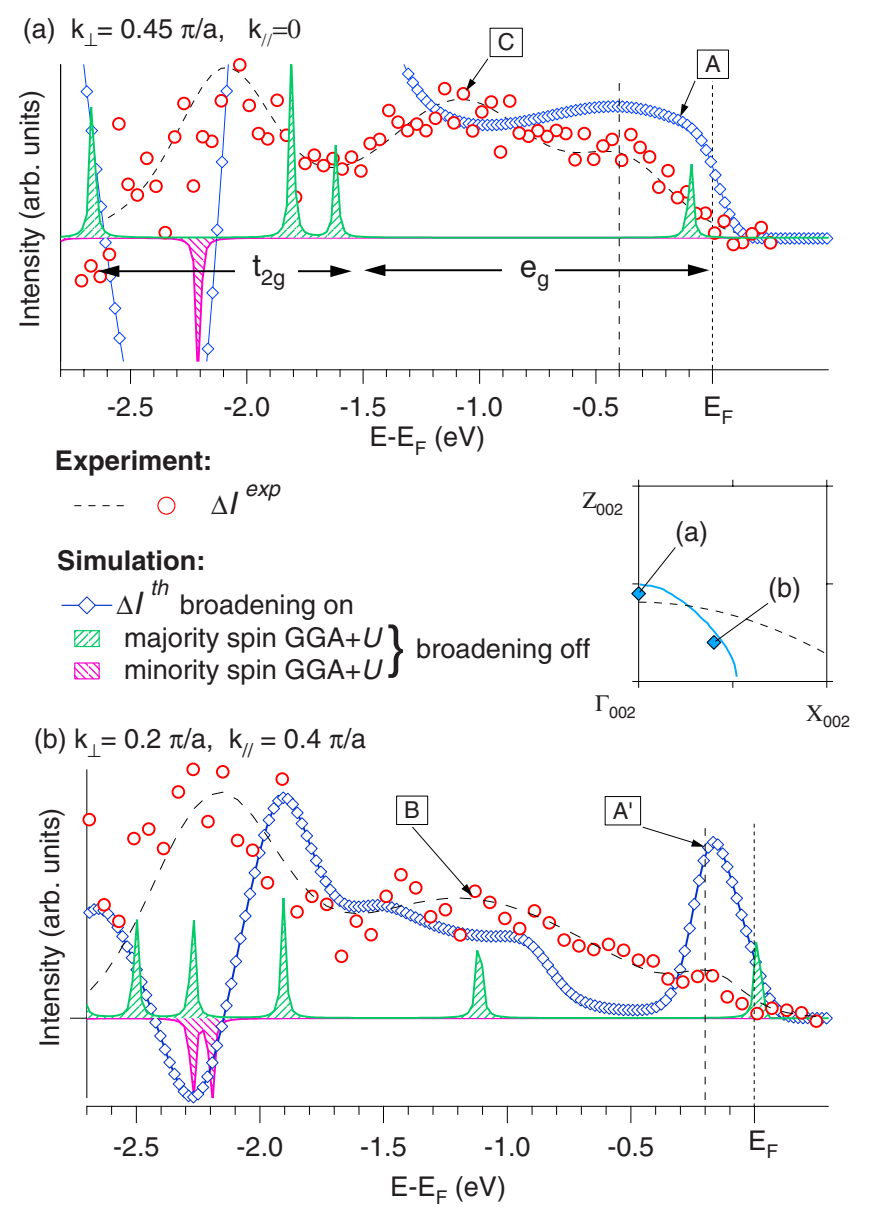

FIG. 8. (Color online) Experimental $I^{\exp }(\bigcirc)$ and simulated $\Delta I^{\text {th }}$ difference spectra with lifetime, $k_{\perp}$ broadening and Gaussian convolution reflecting the experimental resolution of $150 \mathrm{meV}$ (broadening on, $\diamond$ ), compared to the $\Delta I^{\text {th }}$ simulation where the lifetime and $k_{\perp}$ broadening were reduced by a factor of 1000 (broadening off, hatched areas). The peaks $\mathrm{A}^{\prime}, \mathrm{B}$, and $\mathrm{C}$ are discussed in the text. Experimental data for (a) $k_{\|}=0$ and (b) $k_{\|}=0.4 \pi / a$ are compared to simulated spectra where $k_{\perp}$ momenta have maximum intensity at the $E_{F}$ [indicated with $(\diamond)$ in the BZ inset]. The vertical dashed lines below $E_{F}$ are related to the kinks pointed to by arrows in Fig. 3. 
$500 \mathrm{meV}$ found in the data of Chikamatsu et al. ${ }^{24}$ They have interpreted the upward shift of this peak from their $\mathrm{LDA}+U$ calculated conduction band bottom at around $1.3 \mathrm{eV}$, as a signature of band renormalization due to strong electron-phonon coupling or, in our terminology, the FCB peak. However, our ARPES simulations in Fig. 2 reproduce this peak as a signature of $3 \mathrm{D}$ effects originating from the $3 z^{2}-r^{2}$ paraboloid. A characteristic of this origin is the variation of the peak energy with $k_{\perp}$, as illustrated by our ARPES simulations compiled in a movie. ${ }^{17} \mathrm{~A}$ difference of $\sim 100 \mathrm{meV}$ in the peak energy between our data and that of Chikamatsu et al..$^{24}$ can therefore be attributed to a certain difference in $k_{\perp}$ between the two experiments. In fact, their determination of $k_{\perp}$ used empirical free-electron final states with the inner potential optimized for the normal emission, which may become inaccurate when extended to the next BZ (large $\mathbf{K}_{\|}$values). Such non-free-electron effects in the final states are presumably strong in $\mathrm{La}_{2 / 3} \mathrm{Sr}_{1 / 3} \mathrm{MnO}_{3}$ with its highly modulated crystal potential. The FCB structure is, nevertheless, found in our data as peak C. Following the band renormalization approach in Ref. 24, the binding energy of peak $\mathrm{C}$, compared to the $\mathrm{GGA}+U$ calculated conduction band bottom, yields an effective mass renormalization factor of $\approx 2$. Such value is characteristic of a moderately strong electron-phonon coupling, also found in bilayer manganites. ${ }^{26}$

A more elaborate analysis of our data would require taking into account the non-free-electron effects in the final states as well as the matrix element effects, which can be achieved within the one-step photoemission theory (see, for example, Ref. 31 and the references therein). Most time consuming part of this strict approach is computation of the final states described by damped Bloch waves with complex surface-perpendicular $k_{\perp}$. Although this can, in principle, be performed on top of APW + LO computations using $\mathbf{k} \cdot \mathbf{p}$ expansion ${ }^{31}$ such computations still remain hardly feasible for complex materials such as LSMO. This has forced us to restrict our analysis to the free-electron approximation and neglect the matrix element effects.

\section{Determination of $\boldsymbol{k}_{F}$}

The determination of $k_{F}$ along the surface-perpendicular direction $\Gamma Z$ in the BZ is illustrated in Fig. 7(b). The simulation shows that due to $k_{\perp}$ broadening, the Fermi intensity maximum $k_{F}^{\prime}=0.45 \pi / a$ is slightly shifted from the true $k_{F}=0.49 \pi / a$. Using this difference to correct the experimental $k_{F}^{\prime}=0.4 \pi / a$, we arrive at experimental $k_{F}^{\Gamma Z}=0.44 \pi / a$. At first glance, $k_{F}$ along the surface-parallel direction $\Gamma X$ cannot be determined from our data because our photon energy corresponds to the nominal direct transitions at $k_{\perp}^{0}=0.4 \pi / a$ away from the $\Gamma X$ line. However, due to $k_{\perp}$ broadening, the ARPES spectra for any photon energy show nonvanishing Fermi intensity through the whole $k_{\|}$extension of the $3 z^{2}-r^{2}$ paraboloid. $k_{F}$ along the $\Gamma X$ line can then be identified from the drops of Fermi intensity as a function of $k_{\|}$. Note that this method to determine $k_{F}$ appears insensitive to the photon energy. Interestingly, Chikamatsu et al. ${ }^{24}$ arrived at the same method starting from arguments of the FL momentum distribution function $n(\mathbf{k})$.
We have found the Fermi intensity $I_{F}$ by integration within $\pm 150 \mathrm{meV}$ around $E_{F}$. The simulated $I_{F}\left(k_{\|}\right)$dependence and its derivative $\frac{\partial I_{F}\left(k_{\|}\right)}{\partial k_{\|}}$are shown in Fig. 2(f'). The drop in $I_{F}$ identified by the minimum of $\frac{\partial I_{F}\left(k_{k}\right)}{\partial k_{\|}}$is found at $k_{\|}=0.45 \pi / a$, again slightly displaced from the true $k_{F}=0.48 \pi / a$, identified from the peak in $I_{F}$, denoted with $k_{F}$ in Fig. 2( $\left.\mathrm{f}^{\prime}\right)$. The corresponding experimental curve in Fig. $2\left(\mathrm{e}^{\prime}\right)$ differ from the simulation by the suppression of structure $\mathrm{A}^{\prime}$ and larger smearing in $k_{\|}$, which may be traced back to broadening in $\mathbf{k}$ space due to the random nature of LSMO, left away by our calculations based on the VCA. However, the drop in $I_{F}$ is again clearly identified by the minimum of $\frac{\partial I_{F}\left(k_{1}\right)}{\partial k_{\|}}$around $0.42 \pi / a$. Correcting this figure for the displacement from the true $k_{F}$ in the simulations, we arrive at experimental $k_{F}^{\Gamma X}=0.45 \pi / a$. Such a value is in excellent agreement with $k_{F}$ of $0.43-0.48 \pi / a$ found by Chikamatsu et al. ${ }^{24}$ measured at totally different $k_{\perp}^{0}$ corresponding to $h \nu=88 \mathrm{eV}$. Our experimental $k_{F}^{\Gamma Z}$ and $k_{F}^{\Gamma X}$ momenta appear both reduced by $\sim 5 \%$ compared to the GGA $+U$.

\section{B. Spin-resolved ARPES}

The experimental spin-resolved ARPES (SARPES) spectra for $k_{\|}=0$ and $k_{\|}=0.4 \pi / a$ from Fig. 3 are compared with the simulations in Fig. 8. The data are compared in the $I_{\uparrow}-I_{\downarrow}$ representation, with $\Delta I^{\text {exp }}$ denoting experimental and $\Delta I^{\text {th }}$ theoretical $I_{\uparrow}-I_{\downarrow} \cdot{ }^{25}$ The simulations are performed for $k_{\perp}^{0}=k_{F}^{\prime}$, corresponding to the maximum intensity at $E_{F}$ [slightly shifted from the true $k_{F}$, see Fig. 7(a)]. To trace the $k_{\perp}$ broadening and lifetime effects for individual spin channels, we also show spectra simulated with $\delta k_{\perp}$ and lifetime energy broadening reduced by a factor of 1000 . They show sharp peaks sitting at the $\mathrm{GGA}+U$ band energies.

\section{SARPES spectra vs band structure}

For the normal emission [Fig. 8(a)] we find a majority spin $e_{g}$ state close to $E_{F}$. Switching on the $k_{\perp}$-broadening and lifetime effects smears out the $e_{g}$ peak into a hump with a maximum at around $400 \mathrm{meV}(\diamond)$. This energy fits well the position of the kink in the experimental $I_{\downarrow}$ curve [Fig. 3(a)], explaining the sudden reduction in spin polarization around $400 \mathrm{meV}$. More specifically, the energy where the $\Delta t^{\text {th }}$ hump reaches a maximum coincides with the energy where $\Delta I^{\text {exp }}$ reaches a plateau. However, the simulations completely fail to reproduce the experimental feature marked $\mathrm{C}$, which piles up on the plateau at around $1 \mathrm{eV}$ binding energy (BE). It corresponds to feature $\mathrm{C}$ in the experimental spin-integrated spectra in Fig. 2, which we have tentatively ascribed to the FCB effects. The fact of significant polarization coming with this feature corroborates the FCB mechanism, where coupling to the bosonic degrees of freedom does not change the spin. Thus, the incoherent part of the ARPES spectra in Fig. $2(\mathrm{e})$ is around $1 \mathrm{eV}$.

Figure 8(b) summarizes the off-normal $\left(k_{\|}=0.4 \pi / a\right)$ measurements made with the same $h \nu=38 \mathrm{eV}$ as in the normal emission. Extrapolation of the FS map in Fig. 7(a) to this $k_{\|}$ value suggests that these measurements fall into close vicinity of the FS or at least the $k_{\perp}$-broadening interval around it. 


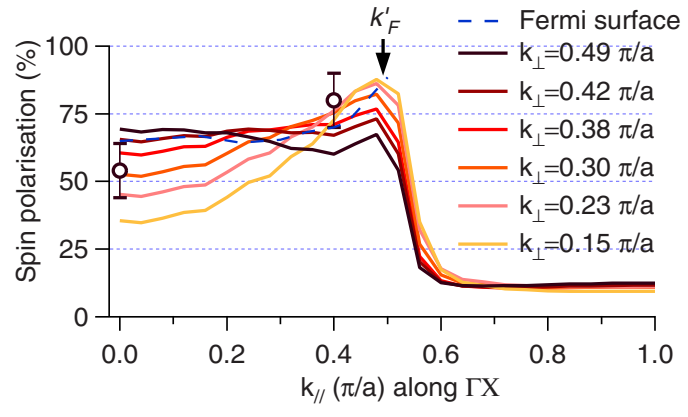

FIG. 9. (Color online) Simulated $k_{\|}$dependence of the spin polarization at $E_{F}$ for different $k_{\perp}$ momenta and at the Fermi surface (dashed line). The simulations assume a Lorentzian tail of the spectral functions up to $E_{F}$. The experimental data $(O)$ are taken from Figs. 3(a) and 3(b).

We therefore compare them with simulations for $k_{\perp}=0.2 \pi / a$ along the curvature of the theoretical FS in Fig. 7(a), also indicated in the BZ inset in Fig. 8. Peak B around $1 \mathrm{eV} \mathrm{BE}$ is smeared due to $k_{\perp}$ broadening, which is well accounted for in the experimental data and simulation. For both $\Delta I^{\text {exp }}$ and $\Delta I^{\text {th }}$, the GGA $+U$ band at $E_{F}$ is smeared due to $k_{\perp}$ broadening. At first glance, this $k_{\perp}$ broadening appears similar to that for $k_{\|}=0$. However, Fig. 4(b) indicates a qualitative difference between the two $k_{\|}$: while $k_{\perp}$ broadening is active in the interior of the whole paraboloid for $k_{\|}=0$, for $k_{\|}=0.4 \pi / a$ it extends towards the ghost feature $\mathrm{A}^{\prime}$ only.

Our spin-resolved data allow us to totally rule out the situation that in $\mathrm{La}_{2 / 3} \mathrm{Sr}_{1 / 3} \mathrm{MnO}_{3}$, the unoccupied minority spin bands are shifted down and reach $E_{F}$. This situation can be described by DFT calculations with $U=0 .{ }^{14}$ First, according to the evaluation in Ref. 3, the expected photoelectron spin polarization at $E_{F}$ in such a case would be only $36 \%$. This value is lower than the measured $55 \%$ beyond the experimental error bars. Second, the calculations with $U=0$ suggest that in this case the bottom of the minority spin bands, found above $E_{F}$ in Fig. 4(a'), would be $\sim 200 \mathrm{meV}$ below $E_{F}$ around the $\Gamma$ point. Although for our normalemission measurements $k_{\perp}$ is away from this point, due to $k_{\perp}$ broadening the minority spin bands would still produce a structure near $E_{F}$ in the spin-down channel. No such structure is seen in our experimental data in Fig. 3. We can therefore exclude the presence of minority spin bands near $E_{F}$.

\section{Spin contrast reduction in SARPES}

However, the most striking features of our spin-resolved ARPES data are the values of polarization at $E_{F}$ less than $100 \%$, yielded by both $k_{\|}=0$ and $k_{\|}=0.4 \pi / a$ spectra. At first sight, this fact indicates that $\mathrm{La}_{2 / 3} \mathrm{Sr}_{1 / 3} \mathrm{MnO}_{3}$ is not a halfmetallic ferromagnet. However, our simulations within the half-metallic picture reproduce well the reduced polarization: Fig. 9 shows the simulated $k_{\|}$dependence of the spin polarization at $E_{F}$ for $k_{\perp}$ following the FS, as well as for different $k_{\perp}$ momenta spanning the BZ. The simulated polarization values of $55 \%$ for $k_{\|}=0$ and $80 \%$ for $k_{\|}=0.4 \pi / a$ match the experiment, especially in view of some uncertainty of $k_{\perp}$ for our off-normal data.
Figure 5 gives a physical picture of such a spin contrast reduction (SCR) effect for the $k_{\|}=0$ case as an example. If the final-state $k_{\perp}$ broadening and initial-state energy broadening both vanish, the PE spectrum is reduced to a $\delta$-function-like peak near $E_{F}$. In our experiment with $h \nu=38 \mathrm{eV}$, this corresponds to the direct transition from the spin-up band at $k_{\perp}=0.4 \pi / a$, which yields $P=100 \%$. With the initial-state lifetime coming into play, the tail of the spectral function $A^{i}(E)$ of the spin-down band at $k_{\perp}=0.4 \pi / a$ extends, in principle, up to $E_{F}$ [ignoring the steep drop in $A^{i}(E)$ at $E_{F}$ (Ref. 15)]. However, its amplitude is insignificant compared to the prominent peak from the spin-up band, and $P$ remains anyway nearly $100 \%$. A dramatic effect comes with $k_{\perp}$ broadening: First, the concomitant averaging within $\delta k_{\perp}$ smears the direct transition peak from the spin-up band, reducing its amplitude much, which is now filling the $k_{\|}$extension of the whole paraboloid [Fig. 4(b)]. Simultaneously, $k_{\perp}$ broadening increases the spectral contribution from the spindown band closer to the $\Gamma$ point, where it moves to lower BE [ $2 \mathrm{eV}$, see Figs. 4( $\left.\mathrm{a}^{\prime}\right)$ and $4\left(\mathrm{~b}^{\prime}\right)$ and Fig. 5], increasing the lifetime tail amplitude near $E_{F}$. The decrease in spin-up contribution and the increase in spin-down one result in the reduction of the spin polarization $P$ observed in the SARPES spectrum. The SCR is thus a combined effect of the finalstate $k_{\perp}$ broadening and initial-state lifetime broadening taking place at the energy scale larger than the $A^{i}(E)$ drop at $E_{F}{ }^{15}$ In our case, the resulting deviation of the spin polarization from $100 \%$ might have been misinterpreted as deviations of $\mathrm{La}_{2 / 3} \mathrm{Sr}_{1 / 3} \mathrm{MnO}_{3}$ from half-metallicity.

For $k_{\|}=0.4 \pi / a$, the SCR effect is less pronounced because with increase in $k_{\|}$, the uppermost spin-down band disperses down [see Fig. 4(b)], reducing the amplitude of the $A^{i}(E)$ tail at $E_{F}$. Figure 9 demonstrates that some uncertainty of $k_{\perp}$ for our off-normal data is not important because the variation of polarization with different $k_{\perp}$ remains comparable with the error bars of the experimental polarization value.

Figure 9 also indicates that the hallmark of $100 \%$ spin polarization is not expected even for other k-space locations along the $\Gamma X$ azimuth. Incorporating the true FL spectral function into our simulations, characterized by the drop in signal near $E_{F},{ }^{15}$ hardly changes our interpretation of the experimental data because our energy resolution of $150 \mathrm{meV}$ is still too large compared to the expected energy width of the drop in signal in the order of $40 \mathrm{meV}$. We conjecture, however, that improvement of the experimental resolution should yield, for the $\mathrm{La}_{2 / 3} \mathrm{Sr}_{1 / 3} \mathrm{MnO}_{3}$ half-metal, spin polarization values approaching $100 \%$ because the lifetime tails in this region readily decay to zero at $E_{F}$.

\section{Ghost features in ARPES and kinks in SARPES}

The simulated SARPES spectra in Fig. 8 reflect intrinsic contributions to the photoemission intensities for noninteracting electrons where (i) the electronic many-body responses to the creation of the photohole and creation of the electron-hole pairs, (ii) matrix element effects, and (iii) extrinsic inelastic processes are not considered. The latter effects occur along the way as photoelectrons propagate toward the surface, and collected energy losses move them 
from the elastic peak position to a smoothly rising inelastic background on the higher BE side underneath the intrinsic spectrum..$^{28}$ On the experimental side, this is well accounted for by a continuous decrease of the polarization $P(E)$ for higher BE, as seen in Figs. 3(a) and 3(b). At the same time, $\Delta I^{e x p}$ in Fig. 8 smears out the elastic spin-down channel of the $t_{2 g}$ states indicated by $\Delta I^{\text {th }}$. Consequently, $P(E, \mathbf{k})$ never reaches negative values, as expected from the sharp $t_{2 g}$ minority spin peaks in Fig. 8.

On the other hand, the $e_{g}$ states below $E_{F}$ are more influenced by the $k_{\perp}$-broadening effects because we find a close correlation between the $\Delta I^{\text {th }}$ maxima (indicated by dashed vertical lines in Fig. 8) and the kinks in Fig. 3. This suggests that the ghost spectral features $\mathrm{A}$ and $\mathrm{A}^{\prime}$ are responsible for the kinks in the experimental $I_{\downarrow}$. In this way, SARPES spectra provide indirect evidence of the ghost spectral feature $\mathrm{A}^{\prime}$ [Fig. 2(e)], which we did not observe in ARPES [Fig. 2(f)]. SARPES made the detection of this edge possible through a spin-selective spectral dependence in a simple way: The contribution of the spin-up electrons from the filled paraboloid exhibits a sudden change compared to flat spin-down lifetime Lorentzian tails in Fig. 4( $\left.b^{\prime}\right)$. Hence, the delayed onsets in $I_{\downarrow}(E, \mathbf{k})$ just mimic a half-gap of no relevance for the band structure.

While the analysis based on $\Delta I^{e x p}$ and $\Delta I^{\text {th }}$ gives a qualitative agreement with the measured polarization $P(E, \mathbf{k})$, the $I_{\uparrow(\downarrow)}(E, \mathbf{k})$ photoemission intensities derived from $P(E, \mathbf{k})$ do not reproduce the simulated $I_{\uparrow(\downarrow)}^{\text {th }}$ signal seen in Fig. 4. In off-normal emission [Fig. 8(b)], the change in $\Delta I^{\text {th }}$ below $E_{F}$, driven by $I_{\uparrow}^{\text {th }}$, is much more pronounced than for $k_{\|}=0$ [Fig. 8(a)]. This is likely the reason why on the experimental side, we observe a small but noticeable shoulder on sloped background in $I_{\uparrow}$ [marked with $\boldsymbol{\Delta}$ in the inset of Fig. 3(b)]. For $k_{\|}=0$, this feature is unresolved [Fig. 3(a)]. In this context, the shoulder is directly related to the spectral feature $\mathrm{A}^{\prime}$. It is not clear, however, why the kink in the $I_{\downarrow}$ channel appears at the same binding energy of $\sim 200 \mathrm{meV}$, suggesting that there is some sort of dependency between the $I_{\uparrow}$ and $I_{\downarrow}$ channels. The same situation is found for $k_{\|}=0$ : The kink in $I_{\downarrow}$ is measured at binding energies right after the $I_{\uparrow}^{\text {th }}$ reaches maximum. These thresholds of 400 and $200 \mathrm{meV}$ are indicated by vertical dashed lines in Figs. 8(a) and 8(b), respectively.

The kinks in $I_{\downarrow}$ channels suggest that we cannot simply use the spin of the electrons as a tag and measure independently the photoelectron spectra for spin-up and spin-down electrons, as assumed within our simulation framework. We conjecture that, together with $k_{\perp}$-broadening effects, a full many-body response of the interacting electrons and electron-hole pairs in a photoemission process is needed to understand the origin of the kinks in the $I_{\downarrow}(E, \mathbf{k})$ spectra.

\section{Angle-integrated spin-resolved photoemission}

An alternative explanation for the kinks in $I_{I}(E, \mathbf{k})$ could be a contribution of unoccupied minority DOS in the inelastic scattering processes. ${ }^{28}$ The kinks would then mark the positions of the bottom of unoccupied minority DOS. Based on the band-structure calculations from Fig. 4( $\left.\mathrm{a}^{\prime}\right)$, however, we would expect the onset in $I_{\downarrow}$ for $k_{\|}=0$ at lower BE than in off-normal emission. Since this is not the case, the coincidence between the kinks and spectral features $\mathrm{A}, \mathrm{A}^{\prime}$ is more realistic. Thus, we tentatively attribute the origin of the kinks in $I_{\downarrow}(E, \mathbf{k})$ to the spin-dependent interplay between the $k_{\perp}$-broadening and many-body effects in PE.

Interestingly, on the experimental side, the angleintegrated spin-resolved measurement from Park et al. ${ }^{2}$ corroborates this picture. By angle integration, the "kinks" of $I_{\downarrow}$ in Fig. 3(b) get smeared out in $k_{\|}$due to the dispersion of feature $\mathrm{A}^{\prime}$ in $k_{\|}$going down to $\sim 800 \mathrm{meV}$ BE [see Fig. 2(f)]. On approaching the $\bar{\Gamma}$ point, $I_{\downarrow}$ starts to pile up due to spectral feature A. According to Fig. 2(f), this happens for $k_{\|}$ $\sim 0.2 \pi / a$, where $\mathrm{A}^{\prime}$ reach $\sim 600 \mathrm{meV} \mathrm{BE}$. Thus, in angleintegrated measurements, the joint effect of $\mathrm{A}$ and $\mathrm{A}^{\prime}$ on $I_{\downarrow}$ suggests a continuous increase of $I_{\downarrow}$ around $600 \mathrm{meV} \mathrm{BE}$, which yields an apparent half-gap of $\sim 600 \mathrm{meV}$, as reported in Ref. 2. We emphasize that the apparent half-gap in Ref. 2, as well as in our data, does not mean that there are no minority spins detected. It is just a measure of the imbalance between the $N^{+}$and $N^{-}$Mott detector counts rendered into absolute spin polarization $P$ via detector calibration.

\section{Half-metallicity: A bulk or surface property?}

According to the universal curve, the probing depth $\lambda$ in our experiment is of the order of $5 \AA$, which in our case is of the order of two $\mathrm{La}_{2 / 3} \mathrm{Sr}_{1 / 3} \mathrm{MnO}_{3}$ unit cells only. This value of $\lambda$ is corroborated by the relevance of the $k_{\perp}$-broadening value used in our simulations. It was speculated (see the review of Nadgorny ${ }^{3}$ and references therein) that the electronic structure within such probing depth does not reflect the bulk properties because of surface effects resulting in overall band narrowing. However, our ARPES results have demonstrated a convincing agreement with the bulk $\mathrm{La}_{2 / 3} \mathrm{Sr}_{1 / 3} \mathrm{MnO}_{3}$ electronic structure described by GGA $+U$, with a $U$ value of $2 \mathrm{eV}$, if the intrinsic broadening mechanisms in the photoemission process are taken into account. For this reason, it is unlikely that the $\mathrm{La}_{2 / 3} \mathrm{Sr}_{1 / 3} \mathrm{MnO}_{3}$ halfmetallicity is just a surface property.

Yet, recent surface $\mathrm{x}$-ray diffraction study on $\mathrm{La}_{2 / 3} \mathrm{Sr}_{1 / 3} \mathrm{MnO}_{3}$ films ${ }^{27}$ have presented a clear experimental evidence that the surface is different from the bulk: The top unit cell layer was characterized by significant $\mathrm{Sr}$ segregation with concentration of $\mathrm{Sr}$ atoms more than twice higher than in the bulk, while the subsurface $\mathrm{La}_{2 / 3} \mathrm{Sr}_{1 / 3} \mathrm{MnO}_{3}$ unit cell layer already had basically the bulk stoichiometry. Thus, our ARPES data reflect Mn $3 d$ photoelectrons from the two topmost unit cells as charge disproportiated $\mathrm{Mn}^{3+} / \mathrm{Mn}^{4+}$ valence states, consistent with bulklike ARPES simulations. Since for Sr concentrations larger than $\sim 35 \%$ the magnetic structure changes to antiferromagnetic, ${ }^{29}$ the suppression of the magnetization near the surface is expected. As a matter of fact, x-ray resonant magnetic stattering spectroscopy demonstrated that magnetization near these interfaces is less than half the bulk value at low temperatures. ${ }^{30}$ Note that it is this reduced surface magnetization that was used as a magnetic quantization axis for our spin-resolved measurement.

The agreement of our experimental data with the $\mathrm{GGA}+U$ bulk band structure seems contradicting, at first 
sight, to the stoichiometric changes near the surface due to enrichment by $\mathrm{Sr}$ atoms. However, the corresponding changes in the electronic structure remain less significant because of the effects discussed above in connection with the $\mathrm{La}-\mathrm{Sr}$ disorder and the similarity of the $\mathrm{La}$ and $\mathrm{Sr}$ scattering potentials.

\section{CONCLUSIONS}

We have reported spin-integrated and spin-resolved ARPES data on 3D compound $\mathrm{La}_{2 / 3} \mathrm{Sr}_{1 / 3} \mathrm{MnO}_{3}$. The measurements were performed in the (100) mirror plane. The spin-resolved measurements were performed in two $\mathbf{k}$-space points characterized by a clear Fermi edge in normal and off-normal emission. The experimental data were interpreted using GGA $+U$ band calculations and simulations of ARPES spectra, taking into account the final-state $k_{\perp}$ broadening and initial-state lifetime broadening intrinsic for the photoemission process.

Due to the 3D nature of $\mathrm{La}_{2 / 3} \mathrm{Sr}_{1 / 3} \mathrm{MnO}_{3}, k_{\perp}$ broadening then results in a plethora of astonishing effects essential for a correct interpretation of the ARPES data: (1) The ARPES signal is formed by adding up contributions from all $k_{\perp}$ within the $k_{\perp}$-broadening interval through the $\mathrm{BZ}$ and does not correspond to the spectral function $A(\omega, \mathbf{k})$ for any fixed $k_{\perp}$. This fundamental fact complicates the analysis of the $A(\omega, \mathbf{k})$ spectral function in 3D systems. (2) The ARPES peaks appear slightly shifted from the $A(\omega, \mathbf{k})$ peaks in both energy and $\mathbf{k}$. For example, the maxima of intensity at $E_{F}$ are found shifted about 5\% from the true Fermi vector. (3) For all photon energies, the whole energy and $k_{\|}$extension of the central paraboloid formed by $3 z^{2}-r^{2} e_{g}$ bands is filled by nonvanishing photoemission intensity with a clear Fermi cut. In particular, the edges of the paraboloid become visible as kinks in the spin-resolved spectra. (4) Values of spin polarization at $E_{F}$ are less than $100 \%$. Despite the apparent contradiction to the half-metallic nature of $\mathrm{La}_{2 / 3} \mathrm{Sr}_{1 / 3} \mathrm{MnO}_{3}$, such a spin contrast reduction finds its explanation in the finalstate $k_{\perp}$-broadening combined with the initial-state lifetime broadening coming into play on the energy scale beyond the QP coherence energy. It should be noted that although — by virtue of embedding the essential mechanisms of the elastic photoemission process-our ARPES simulations have delivered a remarkable understanding of the ARPES data on $\mathrm{La}_{2 / 3} \mathrm{Sr}_{1 / 3} \mathrm{MnO}_{3}$, further quantitative analysis requires rigorous ARPES calculations within the one-step photoemission theory that explicitly accounts for the final state and matrix element effects. ${ }^{31}$

Our spin-resolved data also suggest a strong contribution of inelastic processes to spin polarization, as evidenced by a dramatic decrease in polarization toward higher binding energies. The observed kinks in the $I_{\downarrow}$ spectra mimic a half-gap with energy separation significantly smaller than the true gap in the band structure. Although the exact origin of these kinks remains unclear within the independent particle picture used in our simulations, there is a strong evidence that they are indirectly related to the $k_{\perp}$-broadening effects.

Furthermore, we identify a broad and slightly dispersive ARPES feature around the $\Gamma$ point, which may be explained as due to the Franck-Condon broadening mechanism. This interpretation is corroborated by the spin-resolved data, which show here a peak of polarization because coupling to the bosonic degrees of freedom would not change the spin. Finally, the ARPES simulations taking into account the intrinsic final-state $k_{\perp}$ broadening and initial-state lifetime broadening, have allowed us to reconcile the ARPES data with the bulk band structure of $\mathrm{La}_{2 / 3} \mathrm{Sr}_{1 / 3} \mathrm{MnO}_{3}$ calculated within the $\mathrm{GGA}+U$ framework, without notable flattening or renormalization of the bands, which would otherwise indicate surface effects or correlation effects beyond the $\mathrm{GGA}+U$ picture. In particular, the spin-resolved data are consistent with the half-metallic nature of $\mathrm{La}_{2 / 3} \mathrm{Sr}_{1 / 3} \mathrm{MnO}_{3}$.

\section{ACKNOWLEDGMENTS}

We acknowledge helpful discussions with F. Baumberger (University of St. Andrews), K. Matho (CNRS, Grenoble), M. Grioni (Ecole Polytechnique Fédérale de Lausanne), M. Shi (Paul Scherrer Institut), J. Osterwalder (Physik-Institut, Universität Zürich-Irchel), and J. W. Freeland (Argonne National Laboratory). We are also very grateful to M. Döbeli for a thorough ex situ stoichiometry analysis of the sample using Rutherford backscattering spectrometry. *juraj.krempasky@psi.ch

${ }^{1}$ W. E. Pickett and D. J. Singh, J. Magn. Magn. Mater. 172, 237 (1997).

${ }^{2}$ J.-H. Park, E. Vescovo, H.-J. Kim, C. Kwon, R. Ramesh, and T. Venkatesan, Nature (London) 392, 794 (1998).

${ }^{3}$ B. Nadgorny, J. Phys.: Condens. Matter 19, 315209 (2007).

${ }^{4}$ E. Dagotto, Nanoscale Phase Separation and Collosal Magnetoresistance (Springer-Velag, Berlin, 2003), p. 40.

${ }^{5}$ Y. Tokura and N. Nagaosa, Science 288, 462 (2000).

${ }^{6}$ E. A. Livesay, R. N. West, S. B. Dugdale, G. Santi, and T. Jarlborg, J. Phys.: Condens. Matter 11, 279 (1999).

${ }^{7}$ R. Matzdorf, Appl. Phys. A: Mater. Sci. Process. 63, 549 (1996); V. N. Strocov, J. Electron Spectrosc. Relat. Phenom. 130, 65
(2003); A similar method but neglecting the hole lifetime effects was applied to LSMO in H. Wadati, T. Yoshida, A. Chikamatsu, H. Kumigashira, M. Oshima, H. Eisaki, Z.-X. Shen, T. Mizokawa, and A. Fujimori, Phase Transitions, 79, 617 (2006).

${ }^{8}$ P. R. Willmott, R. Herger, and C. M. Schlepütz, Thin Solid Films 453-454, 436 (2004).

${ }^{9}$ M. Shi, M. C. Falub, P. R. Willmott, J. Krempasky, R. Herger, K. Hricovini, and L. Patthey, Phys. Rev. B 70, 140407(R) (2004).

${ }^{10}$ M. Hoesch, T. Greber, V. Petrov, M. Muntwiler, M. Hengsberger, W. Auwärter, and J. Osterwalder, J. Electron Spectrosc. Relat. Phenom. 124, 263 (2002).

${ }^{11}$ C. Kwon, M. C. Robson, K. C. Kim, J. Y. Gu, S. E. Lofland, S. M. Bhagat, Z. Trajanovic, M. Rajeswari, T. Venkatesan, and A. 
R. Kratz, J. Magn. Magn. Mater. 172, 229 (1997).

${ }^{12}$ P. Blaha, K. Schwarz, G. K. H. Madsen, D. Kvasnicka, and J. Luitz, WIEN2K, an augmented plane wave plus local orbitals program for calculating crystal properties, Vienna University of Technology, Austria, 2001.

${ }^{13}$ D. S. Dessau, T. Saitoh, C.-H. Park, Z.-X. Shen, P. Villella, N. Hamada, Y. Moritomo, and Y. Tokura, Phys. Rev. Lett. 81, 192 (1998).

${ }^{14}$ W. E. Pickett and D. J. Singh, Phys. Rev. B 53, 1146 (1996).

${ }^{15}$ Strictly speaking, the Fermi liquid (FL) properties of the electron system dictate that with an energy approaching the Fermi level, $\operatorname{Im} \Sigma$ reduces to zero. With the FL spectral function having the form $A_{F L}(\mathbf{k}, \omega)=\frac{1}{\pi} \frac{\operatorname{Im} \Sigma(\mathbf{k}, \omega)}{[\omega-E(\mathbf{k})]^{2}+[\operatorname{Im} \Sigma(\mathbf{k}, \omega)]^{2}}, \quad$ where $\quad E(\mathbf{k})=\epsilon_{\mathbf{k}}$ $+\operatorname{Re} \Sigma(\mathbf{k}, \omega)$ is the QP band energy, this property forces $A_{F L}(\mathbf{k}, \omega)$ to drop suddenly to zero at $E_{F}$. The energy scale of this drop is given by the coherence energy $\Delta^{*}$, which is theoretically hardly known. An analysis of the ARPES line shapes for the prototype FL material $\mathrm{TiTe}_{2}$ in Ref. 18 with a causal propagator suggested by Matho (Ref. 19) yields an energy width of the order of $40 \mathrm{meV}$.

${ }^{16}$ A. Goldmann, W. Altmann, and V. Dose, Solid State Commun. 79, 511 (1991).

${ }^{17}$ See EPAPS Document No. E-PRBMDO-77-092812 for a description of the ARPES simulation movie with lifetime and $k_{\perp}$-broadening effects. This document can be reached through a direct link in the online article's HTML reference section or via the EPAPS homepage (http://www.aip.org/pubservs/epaps.html).

${ }^{18}$ S. Harm, R. Dürig, R. Manzke, M. Skibowski, R. Claessen, and J. W. Allen, J. Electron Spectrosc. Relat. Phenom. 68, 111 (1994).

${ }^{19}$ K. Matho, J. Phys. Chem. Solids 56, 1735 (1995); J. Allen, G. Gweon, R. Claessen, and K. Matho, ibid. 56, 1849 (1995).

${ }^{20}$ N. Mannella, W. L. Yang, X. J. Zhou, H. Zheng, J. F. Mitchell, J. Zaanen, T. P. Devereaux, N. Nagaosa, Z. Hussain, and Z.-X.
Shen, Nature (London) 438, 474 (2005).

${ }^{21}$ J. Osterwalder, T. Greber, P. Aebi, R. Fasel, and L. Schlapbach, Phys. Rev. B 53, 10209 (1996).

${ }^{22}$ E. E. Krasovskii (private communication).

${ }^{23}$ D. S. Dessau and Z. X. Shen, in Colossal Magnetoresistive Oxides, edited by Y. Tokura (World Scientific, Singapore, 1998).

${ }^{24}$ A. Chikamatsu, H. Wadati, H. Kumigashira, M. Oshima, A. Fujimori, N. Hamada, T. Ohnishi, M. Lippmaa, K. Ono, M. Kawasaki, and H. Koinuma, Phys. Rev. B 73, 195105 (2006).

${ }^{25}$ We preferred this representation over the conventional polarization because (i) toward higher BE, it suppresses the steep intensity increase seen in the separate $I_{\uparrow}$ and $I_{\downarrow}$ curves in Fig. 3; (ii) above $E_{F}$, it is insensitive to noise; and (iii) $\Delta I^{\text {exp }}$ readily shows counterparts in the $\mathrm{Mn} d_{e_{e}}$ DOS, as depicted in Fig. 8(a).

${ }^{26}$ Z. Sun, Y.-D. Chuang, A. V. Fedorov, J. F. Douglas, D. Reznik, F. Weber, N. Aliouane, D. N. Argyriou, H. Zheng, J. F. Mitchell, T. Kimura, Y. Tokura, A. Revcolevschi, and D. S. Dessau, Phys. Rev. Lett. 97, 056401 (2006).

${ }^{27}$ R. Herger, P. R. Willmott, C. M. Schlepütz, M. Björck, S. A. Pauli, D. Martoccia, B. D. Patterson, D. Kumah, R. Clarke, Y. Yacoby, and M. Döbeli, Phys. Rev. B 77, 085401 (2008).

${ }^{28}$ J. Osterwalder, in Magnetism: A Synchrotron Radiation Approach, Lecture Notes in Physics Vol. 697, edited by E. Beaurepaire, H. Bulou, F. Scheurer, and J.-P. Kappler (SpringerVerlag, Berlin, 2006), p. 106.

${ }^{29}$ G. Banach and W. M. Temmerman, Phys. Rev. B 69, 054427 (2004).

${ }^{30}$ J. W. Freeland, J. J. Kavich, K. E. Gray, L. Ozyuzer, H. Zheng, J. F. Mitchell, M. P. Warusawithana, P. Ryan, X. Zhai, R. H. Kodama, and J. N. Eckstein, J. Phys.: Condens. Matter 19, 1 (2007).

${ }^{31}$ E. E. Krasovskii, V. N. Strocov, N. Barrett, H. Berger, W. Schattke, and R. Claessen, Phys. Rev. B 75, 045432 (2007). 